\title{
PAPER
}

Cite this: Phys. Chem. Chem. Phys., 2021, 23, 17166
Check for updates

\section{Breaking inversion symmetry by protonation: experimental and theoretical NEXAFS study of the diazynium ion, $\mathrm{N}_{2} \mathrm{H}^{+} \dagger$}

\author{
Rafael C. Couto, (D) $\ddagger^{* a}$ Weijie Hua, (D) $*^{b}$ Rebecka Lindblad, (D) cde \\ Ludvig Kjellsson, iD fg Stacey L. Sorensen, (iD c Markus Kubin, iD ${ }^{d}$ Christine Bülow, ${ }^{d}$ \\ Martin Timm, ' Vicente Zamudio-Bayer, iD d Bernd von Issendorff, \\ Johan Söderström, (iD f J. Tobias Lau, (iD dh Jan-Erik Rubensson, ${ }^{f}$ Hans Ågren (iD *fi \\ and Vincenzo Carravetta (iD *j
}

\begin{abstract}
As an example of symmetry breaking in NEXAFS spectra of protonated species we present a high resolution NEXAFS spectrum of protonated dinitrogen, the diazynium ion $\mathrm{N}_{2} \mathrm{H}^{+}$. By ab initio calculations we show that the spectrum consists of a superposition of two nitrogen 1 s absorption spectra, each including a $\pi^{*}$ band, and a nitrogen $1 \mathrm{~s}$ to $\mathrm{H}^{+}$charge transfer band followed by a weak irregular progression of high energy excitations. Calculations also show that, as an effect of symmetry breaking by protonation, the $\pi^{*}$ transitions are separated by $0.23 \mathrm{eV}$, only slightly exceeding the difference in the corresponding dark (symmetry forbidden) and bright (symmetry allowed) core excitations of neutral $\mathrm{N}_{2}$. By DFT and calculations and vibrational analysis, the complex $\pi^{*}$ excitation band of $\mathrm{N}_{2} \mathrm{H}^{+}$is understood as due to the superposition of the significantly different vibrational progressions of excitations from terminal and central nitrogen atoms, both leading to bent final state geometries. We also show computationally that the electronic structure of the charge transfer excitation smoothly depends on the nitrogen-proton distance and that there is a clear extension of the spectra going from infinity to close nitrogen-proton distance where fine structures show some, although not fully detailed, similarities. An interesting feature of partial localization of the nitrogen core orbitals, with a strong, non-monotonous, variation with nitrogen-proton distance could be highlighted. Specific effects could be unraveled when comparing molecular cation NEXAFS spectra, as represented by recently recorded spectra of $\mathrm{N}_{2}^{+}$and $\mathrm{CO}^{+}$, and spectra of protonated molecules as represented here by the $\mathrm{N}_{2} \mathrm{H}^{+}$ion. Both types containing rich physical effects not represented in NEXAFS of neutral molecules because of the positive charge, whereas protonation also breaks the symmetry. The effect of the protonation on dinitrogen can be separated in charge, which extends the high-energy part of the spectrum, and symmetry-breaking, which is most clearly seen in the low-energy $\pi^{*}$ transition.
\end{abstract}

Received 6th May 2021, Accepted 27th July 2021

DOI: $10.1039 / \mathrm{d} 1 \mathrm{cp} 02002 \mathrm{a}$

rsc.li/pccp

\footnotetext{
${ }^{a}$ Department of Theoretical Chemistry and Biology, School of Chemistry, Biotechnology and Health, Royal Institute of Technology, SE-106 91, Stockholm, Sweden. E-mail: rcc@kth.se

${ }^{b}$ MIIT Key Laboratory of Semiconductor Microstructure and Quantum Sensing, Department of Applied Physics, School of Science, Nanjing University of Science and Technology, Nanjing 210094, China. E-mail: wjhua@njust.edu.cn

${ }^{c}$ Department of Physics, Lund University, Box 118, S-22100 Lund, Sweden

${ }^{d}$ Abteilung für Hochempfindliche Röntgenspektroskopie, Helmholtz-Zentrum Berlin für Materialien und Energie, Albert-Einstein-Str. 15, 12489 Berlin, Germany

${ }^{e}$ Inorganic Chemistry, Department of Chemistry - Ångström Laboratory, Uppsala University, SE-75121 Uppsala, Sweden

${ }^{f}$ Department of Physics and Astronomy, Uppsala University, Box 516, SE-75120 Uppsala, Sweden. E-mail: hans.agren@physics.uu.se

${ }^{g}$ European XFEL GmbH, Holzkoppel 4, 22869 Schenefeld, Germany

${ }^{h}$ Physikalisches Institut, Albert-Ludwigs-Universität Freiburg, Hermann-Herder-Str. 3, 79104 Freiburg, Germany

${ }^{i}$ Tomsk State University, 36 Lenin Avenue, Tomsk, Russia

${ }^{j}$ IPCF-CNR, via Moruzzi 1, 56124 Pisa, Italy.E-mail: carravetta@pi.ipf.cnr.it

$\dagger$ Electronic supplementary information (ESI) available: Additional information regarding the theoreticalresults and analysis. See DOI: 10.1039/d1cp02002a

\# Present address: Department of Physics, Stockholm University, Albanova University Center, SE-106 91 Stockholm, Sweden.
} 


\section{Introduction}

Symmetry is exploited in a wide range of physical models. In molecular physics inversion-symmetric molecules have attracted particular interest as the concept of electron localization sometimes seems to be in conflict with the symmetryimposed well-defined parity of the wave functions. ${ }^{1}$ In larger systems dynamic vibronic coupling leads to apparent loss of inversion symmetry, ${ }^{2}$ and in homonuclear diatomics symmetry breaking upon isotope substitution has been investigated. ${ }^{3}$ It has been shown that external fields influence dissociative photoionization of small diatomics, thereby breaking the symmetry. ${ }^{4}$ Also in the field-free case dissociative photoionization leads to symmetry breaking, a general phenomenon which can be understood in terms of entanglement between fragments of well-defined symmetry. ${ }^{5}$ It has been demonstrated that ultrafast core-hole localization in the $\mathrm{N}_{2}$ molecule is reflected in the angular distribution of Auger electrons, ${ }^{6}$ although a description in terms of a coherent superposition of symmetric states is also valid.

The simplicity of near-edge X-ray absorption fine structure (NEXAFS) spectroscopy makes it ideal for a direct investigation of symmetry breaking. For inversion-symmetric molecules dipole selectivity demands parity change in the absorption process, i.e., an ungerade core-excited state must be reached from a gerade ground state. In the NEXAFS spectrum of the $\mathrm{N}_{2}$ molecule the population of the gerade $\pi^{*}$ orbital thus implies the opening of an ungerade core hole. In-line with the dipole selection rule a $\pi^{*}$ resonance that opens the forbidden gerade core hole is not been observed.

Here we explore the role of protonation on NEXAFS spectra by comparing neutral dinitrogen with the diazynium ion, $\mathrm{N}_{2} \mathrm{H}^{+}$. We make use of the new opportunities to measure NEXAFS spectra of molecular ions ${ }^{7-14}$ with high resolution to investigate symmetry breaking in $\mathrm{N}_{2}$ due to protonation. The $\mathrm{N}_{2} \mathrm{H}^{+}$cation (diazynium) has attracted interest due to its astrophysical importance $^{15,16}$ as one of the important components of molecular interstellar clouds, and its spectroscopic constants have been determined to high accuracy, ${ }^{17,18}$ while its neutral parent molecule has not been identified spectroscopically.

We focus here on the analysis of the $\mathrm{N}_{2} \mathrm{H}^{+} \mathrm{X}$-ray absorption spectrum in terms of valence transitions, Rydberg series and continuum resonance transitions, the role of core hole energy splitting and $\mathrm{N}_{2}$ to proton charge transfer transitions, all with respect to nitrogen-proton distance.

Through computational analysis of the energy separation, relative intensity and of different vibrational progressions for the two $\pi^{*}$ states of $\mathrm{N}_{2} \mathrm{H}^{+}$the effect of the proton on the core-excited state of $\mathrm{N}_{2} \mathrm{H}^{+}$in comparison to $\mathrm{N}_{2}$ could be unravelled. Our results indicate that the parity-forbidden gerade core-excitation channel of $\mathrm{N}_{2}$ opens up by protonation in $\mathrm{N}_{2} \mathrm{H}^{+}$. Due to a delicate balance between an electrostatic potential effect and the screening of both core hole and the proton by the electronic cloud, this $\pi^{*}$ splitting is limited to a small value, which, as shown by the analysis presented, still remains distinct from that of $\mathrm{N}_{2}$.

\section{Experiment}

NEXAFS in ion yield mode of the $\mathrm{N}_{2} \mathrm{H}^{+}$molecular cation was measured at the UE52-PGM beamline at the BESSY II synchrotron radiation facility using the Ion Trap end-station. ${ }^{7,19-21}$ The core of this experimental station is a linear radio frequency ion trap cryogenically cooled to low temperatures. Compared to similar measurements obtained in a merged-beam setup,, ${ }^{9,2}$ buffer-gas cooling in a cryogenic ion trap yields cold ions at increased target density and is more likely to give thermalized molecular ions in the electronic ground state.

A mixture of $\mathrm{N}_{2}$ and $\mathrm{H}_{2}$ was ionized in a liquid-nitrogen cooled helium plasma created by a magnetron discharge ion source, where protonated $\mathrm{N}_{2} \mathrm{H}^{+}$was formed. ${ }^{17,23}$ The beam of $\mathrm{N}_{2} \mathrm{H}^{+}$molecular ions was selected by a quadrupole mass filter and further guided to the cryogenic ion trap, which was liquidhelium cooled and aligned with the X-ray beam. Following $\mathrm{X}$-ray absorption, the core excited state relaxes mainly via Auger decay after which the molecular ion dissociates. The resulting product ions, $\mathrm{N}^{+}$and $\mathrm{NH}^{+}$, were also stored in the ion trap, and detected with a reflectron time-of-flight mass spectrometer. Other product ions were not observed: $\mathrm{N}^{q+}(q \geq 2)$ ions are likely to be quenched to $\mathrm{N}^{+}$by collision with helium buffer gas because of the differences in ionization energies. Protons are not trapped at the operating frequencies of the ion trap. X-ray photodissociation action spectra of $\mathrm{N}_{2} \mathrm{H}^{+}$, assumed to be proportional to the $\mathrm{X}$-ray absorption spectrum, were recorded by monitoring the $\mathrm{N}^{+}$and $\mathrm{NH}^{+}$product ion yield while scanning the photon energy. These different product ions gave very similar ion yield spectra.

The ion yield spectra were measured with different settings for the 400-404 eV photon energy region and for the photon energy region above $404 \mathrm{eV}$. These low (high) energy regions were measured with a step size of $10 \mathrm{meV}$ (50 meV) and a beamline exit slit of $20 \mu \mathrm{m}(50 \mu \mathrm{m})$ giving an energy resolution of $50 \mathrm{meV}(75 \mathrm{meV})$. The $\pi^{*}$ resonance of neutral $\mathrm{N}_{2}$ was measured in a gas-cell with a step size of $8 \mathrm{meV}$ and a beamline exit slit of $20 \mu \mathrm{m}$. The measurement of neutral $\mathrm{N}_{2}$ serves as a comparison to the nitrogen edge of $\mathrm{N}_{2} \mathrm{H}^{+}$, but also to crosscheck the photon energy calibration ${ }^{24}$ that was performed on the neon 1s excitation in different reflection orders of the monochromator grating. The photon energy uncertainty at $400 \mathrm{eV}$ is estimated as better than $50 \mathrm{meV}$.

\section{Theory}

\subsection{Electronic structure}

The $\mathrm{N}_{2} \mathrm{H}^{+}$is a linear molecule in its ground state; ${ }^{17,25,26}$ submillimeter-wave spectroscopy determines the equilibrium distances to $R_{\mathrm{N}_{\mathrm{a}}-\mathrm{N}_{\mathrm{b}}}=1.09369 \AA$ and $R_{\mathrm{N}_{\mathrm{b}}-\mathrm{H}}=1.03460 \AA$ 只, ${ }^{18}$ where $\mathrm{N}_{\mathrm{a}}$ and $\mathrm{N}_{\mathrm{b}}$ refer here, and in the following, to the terminal and the central nitrogen atom, respectively. The most accurate coupled cluster calculations $(\operatorname{CCSD}(\mathrm{T})$ with a $\operatorname{TZ2P}(\mathrm{f}, \mathrm{d})$ basis set for $\mathrm{N}_{2} \mathrm{H}^{+}$available in the literature ${ }^{27}$ confirm a linear geometry with $R_{\mathrm{N}_{\mathrm{a}}-\mathrm{N}_{\mathrm{b}}}=1.096 \AA$ and $R_{\mathrm{N}_{\mathrm{b}}-\mathrm{H}}=1.031 \AA$. Comparable values have been obtained by our present RASPT2 ${ }^{28}$ calculation with 
ANO-RCC-VQZP basis set ${ }^{29}: R_{\mathrm{N}_{\mathrm{a}}-\mathrm{N}_{\mathrm{b}}}=1.096 \AA$ and $R_{\mathrm{N}_{\mathrm{b}}-\mathrm{H}}=1.033 \AA$. Reported $\mathrm{HF}^{25}$ and our DFT calculations also reproduce the equilibrium geometry of $\mathrm{N}_{2} \mathrm{H}^{+}$and show that the linear geometry is maintained when the $\mathrm{N}_{\mathrm{b}}-\mathrm{H}$ bond is stretched up to breaking. The HF dissociation energy is about $119 \mathrm{kcal} \mathrm{mol}^{-1}$, comparable to the $\operatorname{CCSD}(\mathrm{T})$ value of $123.7 \mathrm{kcal} \mathrm{mol}^{-1} .^{27}$

The electronic structure of the core excited states of $\mathrm{N}_{2} \mathrm{H}^{+}$ was studied by two ab initio methods with different characteristics: the static-exchange (STEX) ${ }^{30}$ independent particle approximation and the state-average restricted active space self-consistent field (RASSCF) $^{31-33}$ method, followed by a multi-state second-order perturbation (MS-RASPT2), ${ }^{28}$ as implemented in the OpenMolcas software. ${ }^{34}$ In both cases the quasi-stationary core-excited states were optimized by imposing core-valence separation in order to avoid the variational collapse due to filling of the core orbital. ${ }^{35,36}$

The first method does not include electronic correlation but completely describes electronic relaxation and makes it possible to use an almost complete basis set, providing a description of the entire spectrum both below the ionization threshold and in the continuum. For the HF calculations, performed by using the DALTON code ${ }^{37}$, the cc-pVQZ basis $\operatorname{set}^{38,39}$ was employed, augmented by (25s, 25p, 10d) uncontracted gaussians, with even tempered exponents, on the excitation site in STEX calculations.

The second method (RASSCF) treats electron correlation explicitly but can be practically applied only up to the lowest Rydberg excitations. For these calculations the ANO-RCC-VQZP basis set ${ }^{29}$ along with an auxiliary (8s6p4d) Rydberg basis set was used. The Abelian point group $C_{2 \mathrm{v}}$ was used. Scalar relativistic effects have been included by using a second-order Douglas-KrollHess Hamiltonian. ${ }^{40,41}$ In the RASPT2 step, an imaginary shift of 0.3 Hartree $^{42}$ and the default ionization-potential electron-affinity (IPEA) shift of 0.25 Hartree $^{43}$ were in use. The transition dipole moments in the velocity gauge were obtained by the RAS stateinteraction approach. ${ }^{44,45}$ The results presented in Fig. 1 and Table 2 were obtained by considering an active space configuration which involves the use of all three RAS1, RAS2 and RAS3 spaces. The two nitrogen 1s orbitals were placed in the RAS1 space, where a maximum of one hole was allowed, and kept frozen in order to avoid variational collapse. The occupied $3 \sigma, 4 \sigma, 5 \sigma$ and the two $1 \pi$ orbitals were placed in the RAS2 space. A total of 20 unoccupied orbitals were included in the RAS3 space, where a maximum occupation of three electrons was allowed. This approach was successfully applied to other molecular ions, ${ }^{12,13}$ and extra details of the active space scheme and underlying analysis can be found in Couto et al. ${ }^{13}$

\subsection{Vibrational analysis}

To further analyze the fine structure of the experimental nitrogen 1s $\rightarrow \pi^{*}$ band of $\mathrm{N}_{2} \mathrm{H}^{+}$around $402 \mathrm{eV}$ (see Fig. 1), vibrationally resolved XAS spectrum was simulated using a locally modified version of the DynaVib package ${ }^{46}$ interfaced to the GAMESS$\mathrm{US}^{47,48}$ package for electronic structure calculations. We chose

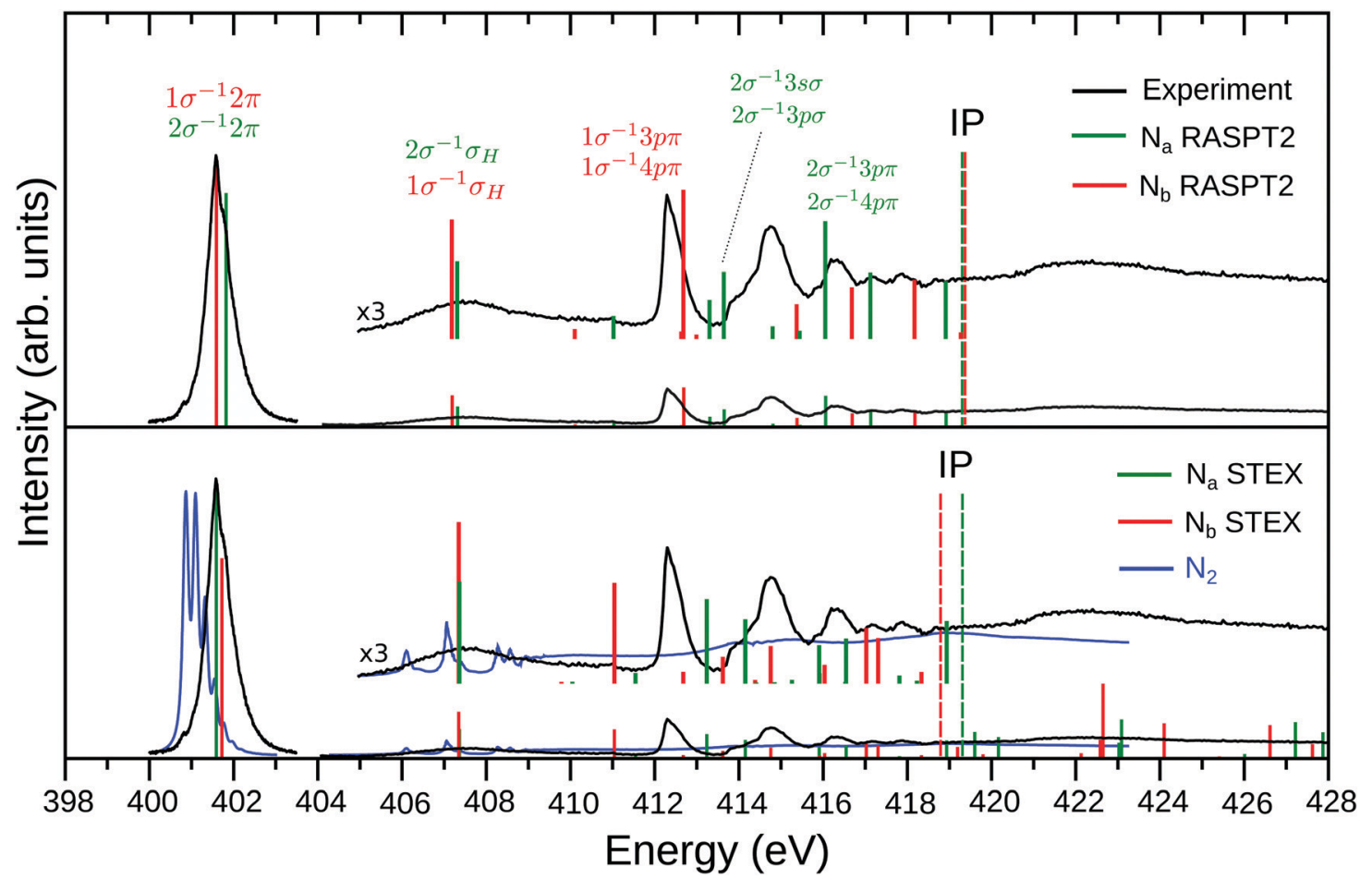

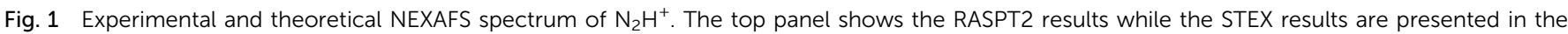

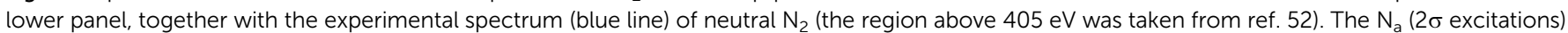

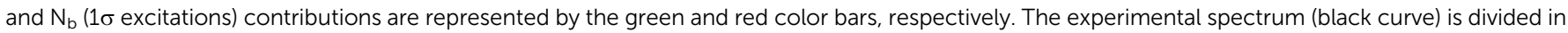

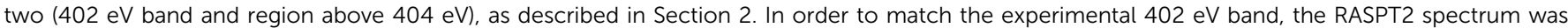

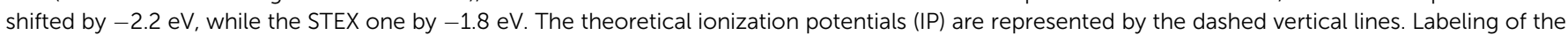
final states above $413 \mathrm{eV}$ simply refers to the main single excitation component of the transition; see Table 2 for more details. 
the DFT method with the BLYP functional ${ }^{49}$ for the calculations. In the ground state, this cation has two degenerate $\pi^{*}$ orbitals $\pi_{x z}{ }^{*}$ and $\pi_{y z} z^{*}$. Since they give equivalent contributions in a orientationally-averaged measurement, our vibronic analysis was only performed for one of these states, say, $\pi_{x z}{ }^{*}$. A doublebasis set technique ${ }^{50}$ was also adopted (Table S2, ESI $\dagger$ ). The protocol was recently verified to produce accurate vibrationallyresolved X-ray photoelectron spectroscopy (XPS) at the C1s edge for a series of cyclic molecules. ${ }^{50}$ For the purpose of validation such simulation was first performed for $\mathrm{N}_{2}$ with various density functionals. The Franck-Condon (FC) factors were simulated by using the Duschinsky rotation (DR) method. ${ }^{51} \mathrm{~N}_{2}$ provides a good benchmark because the vibrationally-resolved band $1 \mathrm{~s} \rightarrow \pi^{*}$ of this molecule is known with high experimental resolution. It turned out that BLYP density functional provides the best agreement with the experimental spectrum and thus such functional was adopted for studying $\mathrm{N}_{2} \mathrm{H}^{+}$too.

Special treatment was done for $\mathrm{N}_{2} \mathrm{H}^{+}$whose ground state geometry is linear while there is no linear minimum for the $1 \mathrm{~s}$ $\mathrm{XCH}$ state of either nitrogen. Only a linear saddle point (minima along the two stretching modes and the bending mode in the $y z$ plane, maxima along the bending mode in the $x z$ plane, see Fig. S4, ESI $\dagger$ ) exists. We had scanned the potential energy surfaces (PESs) along the $x z$ bending mode, which turned out to be of double-well shape, and solved the onedimensional Shrödinger equation numerically to get the FC factors along this mode. More computational details for the vibrational fine structures are provided in the ESI. $\dagger$

A Lorentzian line shape with half-width at half-maximum (HWHM) of $\Gamma_{\mathrm{L}}=0.0565 \mathrm{eV}$ (in accord with the experimental lifetime of $\mathrm{N}_{2}{ }^{+12}$ ) was employed to convolute the stick spectrum of both $\mathrm{N}_{2}$ and $\mathrm{N}_{2} \mathrm{H}^{+}$obtained by the vibrational energy levels and the corresponding Franck-Condon factors. For $\mathrm{N}_{2} \mathrm{H}^{+}$, an additional Gaussian broadening with HWHM of $\Gamma_{\mathrm{G}}=0.07 \mathrm{eV}$ was applied in order to better compare with experimental profile (that is, a Voigt brodening). Finally, ad hoc uniform shifts of 1.10 and $1.23 \mathrm{eV}$ were applied to the theoretical spectra of $\mathrm{N}_{2}$ and $\mathrm{N}_{2} \mathrm{H}^{+}$spectra to better compare with corresponding experiments.

\section{Results}

\subsection{Overview}

An overview of the experimental results for $\mathrm{N}_{2} \mathrm{H}^{+}$is shown in Fig. 1 (black lines). The low energy region of the well-known NEXAFS spectrum of $\mathrm{N}_{2}$ (blue line in the lower panel of Fig. 1) is dominated by the excitation from the nitrogen core level to the lowest unoccupied orbital (LUMO), i.e., the $1 \sigma_{\mathrm{u}}{ }^{-1} 1 \pi_{\mathrm{g}}$ excitation band centered at $401.7 \mathrm{eV}$ commonly referred to as the $\pi^{*}$ resonance. At higher energies the Rydberg series converge to the ionization threshold around $410 \mathrm{eV}$, followed by double excitations and a shape resonance in the $412-420 \mathrm{eV}$ range, ${ }^{52}$ see also Fig. 4 . The extra proton potential in $\mathrm{N}_{2} \mathrm{H}^{+}$extends the $\mathrm{N}_{2}$ spectrum roughly by a factor of two.

The general appearance of the NEXAFS spectrum of the protonated molecule $\mathrm{N}_{2} \mathrm{H}^{+}$shows similarity with the $\mathrm{N}_{2}$ spectrum; it is dominated by a $\pi^{*}$ band that is shifted by approx. $1 \mathrm{eV}$ towards higher energies compared to $\mathrm{N}_{2}$. The finestructure of this resonance is modified in the $\mathrm{N}_{2} \mathrm{H}^{+}$spectrum as analyzed in detail below. The features at higher energies show a complex pattern below the ionization limits, which are theoretically estimated to be around $420 \mathrm{eV}$ (see Fig. 1). The higherenergy features are more intense, relative to the $\pi^{*}$ resonance, than in the $\mathrm{N}_{2}$ spectrum, and the trend is that the sharp spectral structures shift to higher energies with the ionization limits. A salient difference between the $\mathrm{N}_{2} \mathrm{H}^{+}$and $\mathrm{N}_{2}$ spectra, see also Fig. 4 , is the appearance of an extra band in the former spectrum, in between the $\pi^{*}$ transition and Rydberg progressions. As firmly established below, this band originates in a charge transfer of a nitrogen core electron, terminal or central, to the proton, as was already predicted ${ }^{26}$ for $\mathrm{N}_{2} \mathrm{H}^{+}$valence shell excitations.

As the $\mathrm{N}_{2}{ }^{+}$ion has a vacancy in the $3 \sigma_{\mathrm{g}}$ orbital a dominating feature in the NEXAFS spectrum of $\mathrm{N}_{2}{ }^{+}$is due to the $1 \sigma_{\mathrm{u}} \rightarrow 3 \sigma_{\mathrm{g}}$ transition. ${ }^{12}$ No counterpart to this transition is found in the NEXAFS spectrum of $\mathrm{N}_{2} \mathrm{H}^{+}$in Fig. 1; this experimental evidence suggests that there is no open $5 \sigma$ orbital in $\mathrm{N}_{2} \mathrm{H}^{+}$.

\subsection{Nitrogen-proton distance dependence of $\mathrm{N}_{2} \mathrm{H}^{+}$electronic properties}

Based on the previous observations, the HF description of the ground state may be considered valid, even when $R_{\mathrm{N}_{\mathrm{b}}-\mathrm{H}}$ varies over a large range. It is interesting to consider the variation of selected energetical (see Fig. 2) and structural (see Fig. 3) properties of the $\mathrm{N}_{2} \mathrm{H}^{+}$system with the distance of the proton from the $\mathrm{N}_{2}$ fragment.

By HF geometry optimization of the cation at different fixed $R_{\mathrm{N}_{\mathrm{b}}-\mathrm{H}}$ values the potential (black) curve in Fig. 2 is obtained; in agreement with the potential ${ }^{25}$ previously obtained keeping

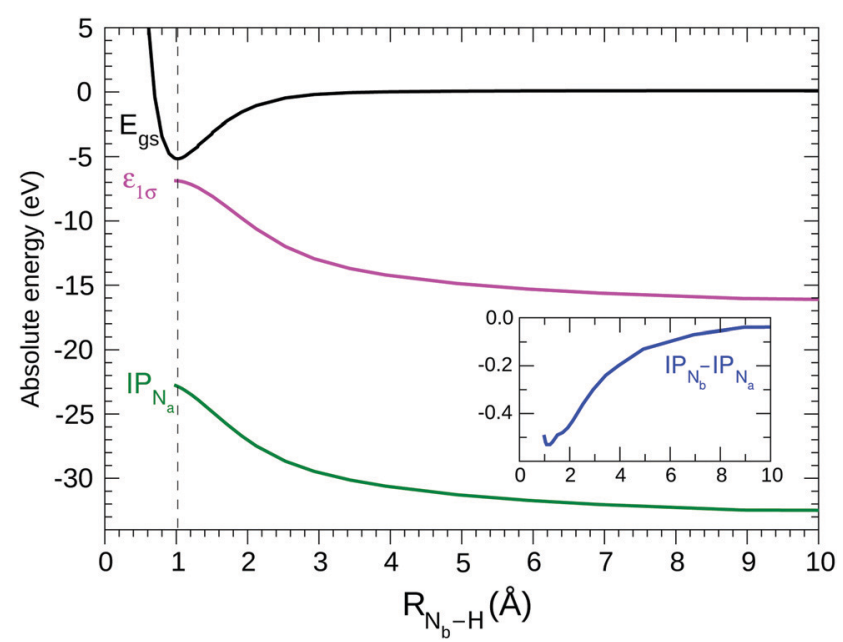

Fig. 2 Energy properties of $\mathrm{N}_{2} \mathrm{H}^{+}$vs the distance $\mathrm{N}_{\mathrm{b}}-\mathrm{H}$ computed at the HF level: ground state energy (black line); lowest frozen core ionization potential according to Koopmans' theorem (KT) (magenta line); relaxed core ionization potential IP of atom $\mathrm{N}_{\mathrm{a}}$ (green line); in the inset: difference between $\mathrm{N}_{b}$ and $\mathrm{N}_{\mathrm{a}}$ core ionization potentials; all the ionization potential values have been shifted by $-444 \mathrm{eV}$, while the gs energy has been shifted by $+2960 \mathrm{eV}$. The equilibrium value of $R_{\mathrm{N}_{\mathrm{b}}-\mathrm{H}}$ is $1.0 \AA$, indicated by the vertical dashed line. 
rigid the $\mathrm{N}_{2}$ fragment. Our $\mathrm{HF}$ calculations confirm, ${ }^{25}$ see Fig. 2, that the cation dissociates in the $\mathrm{N}_{2}$ and $\mathrm{H}^{+}$fragments, as could be expected considering that the ionization potential of the $\mathrm{N}_{2}$ molecule $(15.6 \mathrm{eV})$ is larger than that of $\mathrm{H}(13.6 \mathrm{eV})$. The contribution of the $\left[\mathrm{N}_{2}^{+}+\mathrm{H}\right]$ electronic configuration appears negligible at a great distance of $\mathrm{H}$ from $\mathrm{N}_{2}$, although it slightly contributes to the ground state wave function at the equilibrium geometry.

From Fig. 3 it can be seen (black line in the top graph) that the $R_{\mathrm{N}_{\mathrm{a}}-\mathrm{N}_{\mathrm{b}}}$ bond length at the approach of the proton remains constant down to about $R_{\mathrm{N}_{\mathrm{b}}-\mathrm{H}}=3 \AA$, then decreases until the equilibrium geometry where it takes on a value not very different from that of the $\mathrm{N}_{2}$ molecule. The presence of the proton has a larger effect on the electron density distribution, ${ }^{26}$ as shown in the middle graph of Fig. 3, which reports the variation of the electron charge around the proton and around the two $\mathrm{N}$ atoms estimated by the atomic Mulliken population. Starting from a zero value at a large distance, the proton begins to subtract electronic charge from $\mathrm{N}_{2}$ below $3 \AA$ of distance. From this point towards the equilibrium geometry, the accumulation of electronic charge on $\mathrm{H}$ proceeds uniformly but with a strong polarization effect of the charge on the two nitrogen atoms. Initially it is the $\mathrm{N}_{\mathrm{a}}$ atom that preferably gives up electronic charge to $\mathrm{H}$ and also to the central $\mathrm{N}$ atom, while at a further approach to the equilibrium geometry it is the $\mathrm{N}_{\mathrm{b}}$ atom that provides the greatest contribution. The protonation of $\mathrm{N}_{2}$ leads to the formation of a strong and peculiar $\mathrm{N}-\mathrm{H}$ bond as the bonding electron charge is provided by the molecular fragment alone, in particular by the mixing of the $2 \sigma_{\mathrm{u}}$ orbital with the $1 \mathrm{~s}$ hydrogenlike orbital. This polarization and then decrease of the electron charge in the $\mathrm{N}_{2}$ fragment is related to the symmetry breaking by protonation which becomes very evident in the electronic

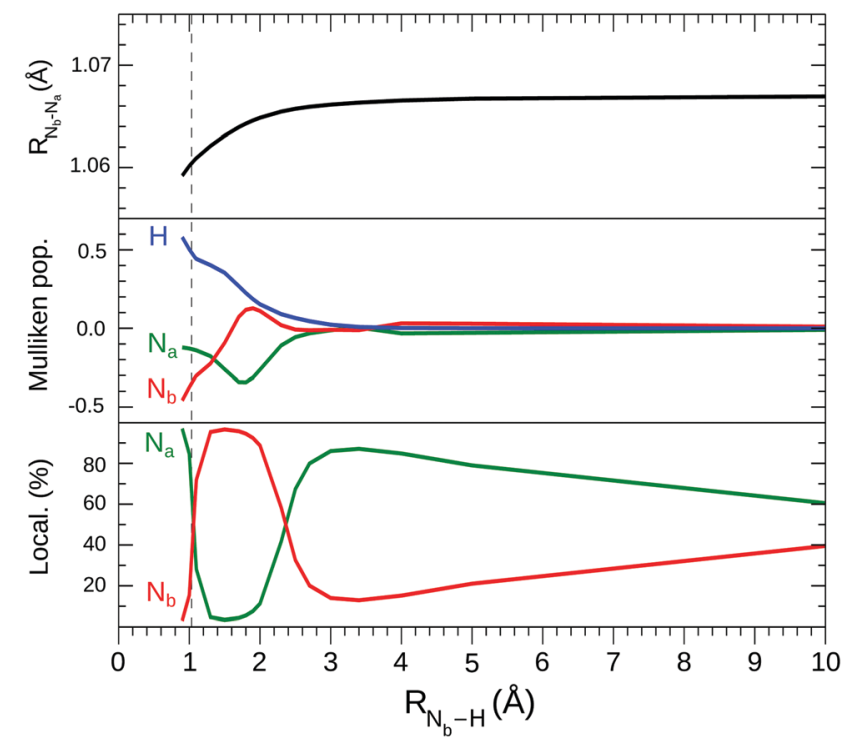

Fig. 3 Structure properties of $\mathrm{N}_{2} \mathrm{H}^{+}$vs the distance $\mathrm{N}_{\mathrm{b}}-\mathrm{H}$ computed at the HF level; top: $\mathrm{N}-\mathrm{N}$ bond length; middle: variation of electron Mulliken population; bottom: localization of the lowest core orbital on the $\mathrm{N}_{\mathrm{a}}$ and $\mathrm{N}_{\mathrm{b}}$ atoms. The equilibrium value of $R_{\mathrm{N}_{\mathrm{b}}-\mathrm{H}}$ is $1.0 \AA$, indicated by the vertical dashed line. structure of the two core orbitals. The bottom graph of Fig. 3 shows the percentage localization of the "gerade like" core orbital around the two $\mathrm{N}$ nuclei vs the proton distance; this quantity is qualitatively estimated by the largest expansion coefficients of the core molecular orbital on the atomic basis functions of $\mathrm{s}$ type. Starting from a large $R_{\mathrm{N}_{\mathrm{b}} \mathrm{H}}$ distance of $>100 \AA$, where delocalization is complete, there is, decreasing the proton distance, an initial slow increase in localization followed by a quite peculiar variation, at short distances, due to the complex interaction of the $\sigma$ orbitals (including the core orbitals) with the proton. Finally, at the equilibrium geometry, the $1 \sigma$ and $2 \sigma$ orbitals localize mostly on $\mathrm{N}_{\mathrm{a}}$ and $\mathrm{N}_{\mathrm{b}}$, respectively.

\subsection{Nitrogen core orbitals}

In light of these findings it is interesting to look at the $\mathrm{N}_{2} \mathrm{H}^{+}$ cation as formed by the two blocks $\mathrm{N}_{2}$ and $\mathrm{H}^{+}$and consider, in comparison with the $\mathrm{N}_{2}$ molecule, the influence of the proton on the core electronic structure, and in particular to evaluate the relevance of the electrostatic interaction and the effect of the symmetry breaking from $D_{\infty \mathrm{h}}$ of $\mathrm{N}_{2}$ to $C_{\infty \mathrm{v}}$ of the fragment $\mathrm{N}_{2}$ in $\mathrm{N}_{2} \mathrm{H}^{+}$. In $\mathrm{N}_{2}$ the core orbitals are perfectly delocalized and have well-defined parity. They can be described, in terms of atomic orbitals, as $1 \sigma_{\mathrm{g}}=\left(+0.70711 \mathrm{~s}_{\mathrm{N}_{\mathrm{a}}}+0.70711 \mathrm{~s}_{\mathrm{N}_{\mathrm{b}}}\right)$ and $1 \sigma_{\mathrm{u}}=\left(-0.70711 \mathrm{~s}_{\mathrm{N}_{\mathrm{a}}}+0.70711 \mathrm{~s}_{\mathrm{N}_{\mathrm{b}}}\right)$. Inversion symmetry is broken in $\mathrm{N}_{2} \mathrm{H}^{+}$, the two sites become nonequivalent and localization is reflected in the magnitude of the expansion coefficients, see Fig. 3. In the ground state of $\mathrm{N}_{2} \mathrm{H}^{+}$at the equilibrium geometry the two core orbitals tend to localize, although not perfectly: $1 \sigma=\left(+0.86041 \mathrm{~s}_{\mathrm{N}_{\mathrm{a}}}+0.50941 \mathrm{~s}_{\mathrm{N}_{\mathrm{b}}}\right)$, $2 \sigma=\left(-0.50961 \mathrm{~s}_{\mathrm{N}_{\mathrm{a}}}+0.86091 \mathrm{~s}_{\mathrm{N}_{\mathrm{b}}}\right)$. Via the sign of the expansion coefficients, the $1 \sigma$ and $2 \sigma$ orbitals of $\mathrm{N}_{2} \mathrm{H}^{+}$are related to the parity constrained $1 \sigma_{\mathrm{g}}$ and $1 \sigma_{\mathrm{u}}$ orbitals of $\mathrm{N}_{2}$, respectively.

The energy spacing of the core orbitals is $0.133 \mathrm{eV}$ in $\mathrm{N}_{2} \mathrm{H}^{+}$, which can be compared to $0.102 \mathrm{eV}$ in $\mathrm{N}_{2}$. In the KT approximation the two core ionization potentials have values $\mathrm{IP}_{1 \sigma}=$ $437.10 \mathrm{eV}$ and $\mathrm{IP}_{2 \sigma}=436.97 \mathrm{eV}$ (see Table 1), while the lowest virtual orbitals $2 \pi\left(\pi^{*}\right)$ have energy $-4.75 \mathrm{eV}$ and can be related to the $1 \pi_{\mathrm{g}}$ orbitals of $\mathrm{N}_{2}$. Neglecting electronic relaxation is a coarse approximation since the relaxation around the core hole is very rapid. However, if we introduce electronic relaxation without localizing the core orbitals, i.e. keeping the mixing observed in the ground state core orbitals, we obtain $\mathrm{IP}_{1 \sigma}=$ $430.03 \mathrm{eV}$ and $\mathrm{IP}_{2 \sigma}=429.88 \mathrm{eV}$ with a difference of $0.15 \mathrm{eV}$ very similar to that of the KT values but with absolute values still very far away from the ionization thresholds that can be guessed from the experimental spectrum in Fig. 1. Furthermore, using the two relaxed but not localized $\sigma$ orbitals, we obtain (in parenthesis: excitation-energy (eV)/oscillator-strength): $1 \sigma \rightarrow 2 \pi^{*}(412.53 /$ $0.001)$ and $2 \sigma \rightarrow 2 \pi^{*}(412.41 / 0.092)$, i.e., the transition from $1 \sigma$, has around $0.12 \mathrm{eV}$ higher energy than transitions from $2 \sigma$, and gets very little intensity. The situation is similar to the $\mathrm{N}_{2}$ case, where the excited state $1 \sigma_{\mathrm{g}} \rightarrow 1 \pi_{\mathrm{g}}$ is energy shifted relative to the excited state $1 \sigma_{\mathrm{u}} \rightarrow 1 \pi_{\mathrm{g}}$ by around $0.12 \mathrm{eV}$, and it is dipole forbidden due to parity.

By allowing complete electronic relaxation around the localized holes, the $\pi^{*}$ excitation energies are in much better agreement 
Table 1 Theoretical core-LUMO excitation energy $\left(E_{\text {exc }}\right)$ and core ionization energy $\left(E_{\text {ion }}\right)$ at nitrogen atom $(\mathrm{N})$ of $\mathrm{N}_{2}$ molecule and nitrogen atoms $\left(\mathrm{N}_{\mathrm{a}}, \mathrm{N}_{\mathrm{b}}\right)$ of $\mathrm{N}_{2} \mathrm{H}^{+}$in different $\mathrm{HF}$ approximations: frozen orbitals, relaxed and delocalized orbitals (Relax-deloc), relaxed and localized orbitals (Relax-local)

$$
\underline{E_{\text {exc }}(\mathrm{eV})} \frac{E_{\text {ion }}(\mathrm{eV})}{}
$$

Atom Frozen Relax-deloc Relax-local Frozen Relax-deloc Relax-local

$\begin{array}{lllllll}\mathrm{N} & & & 402.43 & & & 410.16 \\ \mathrm{~N}_{\mathrm{a}} & 432.35 & 412.53 & 403.38 & 437.10 & 430.03 & 421.07 \\ \mathrm{~N}_{\mathrm{b}} & 432.22 & 412.41 & 403.52 & 436.97 & 429.88 & 420.56\end{array}$

with the experiment; we obtain two "bright" core excited states: $1 \sigma \rightarrow \pi^{*}(403.38 / 0.058) 2 \sigma \rightarrow \pi^{*}(403.52 / 0.042)$ (see Table 1$)$. The two transitions collect more or less the intensity of the allowed transition in the case of delocalized holes, with some increase due to the further contraction of the orbitals due to complete electronic relaxation. In this sense, symmetry breaking and core hole localization in $\mathrm{N}_{2} \mathrm{H}^{+}$makes the "dark" $1 \sigma_{\mathrm{g}} \rightarrow 1 \pi_{\mathrm{g}}$ excited state of the " $\mathrm{N}_{2}$ fragment" visible by borrowing intensity from the "bright" $1 \sigma_{\mathrm{u}} \rightarrow 1 \pi_{\mathrm{g}}$ excited state. Finally we may observe that the trend for the ionization potentials $(\Delta \mathrm{IP}$ line in the inset of Fig. 2), obtained by forcing the localization of the core holes, is quite regular and clearly shows how the presence of the proton separates the core IPs at the equilibrium geometry.

We add that all these features have been confirmed by RASSCF and DFT calculations, which differ from the HF prediction only in details. With respect to the energy separation of the two $\pi^{*}$ excitations, we indeed find differences between the theoretical approximations, but only in terms of variations of order of $0.1 \mathrm{eV}$, see summary Tables S1 and S2 in ESI. $\dagger$ Our best RASPT2 calculation, see Table 2, predicts a vertical energy difference of about $0.23 \mathrm{eV}$, while DFT calculations give a difference of about $0.3 \mathrm{eV}$ (Table S3 of ESI $\dagger$ ). This confirms a symmetry breaking as these values exceeds the corresponding difference between the bright and dark $\pi^{*}$ states of $\mathrm{N}_{2}(0.10 \mathrm{eV})$. Furthermore, the intensity ratio $\left(f_{\mathrm{N}_{\mathrm{a}}} / f_{\mathrm{N}_{\mathrm{b}}}\right)$ of 0.71 from DFT, which is quite stable with the considered functionals, and of about 0.88 from RASPT2 (with more variation with the active space and basis set) is evidently quite different from zero.

\subsection{Computational analysis of the nitrogen-proton distance dependence of the $\mathrm{N}_{2} \mathrm{H}^{+}$photoabsorption spectrum}

To connect the $\mathrm{N}_{2} \mathrm{H}^{+}$and $\mathrm{N}_{2}$ NEXAFS spectra, the transition from dinitrogen to diazynium was analyzed computationally.

The NEXAFS spectrum was first calculated for various $R_{\mathrm{N}_{\mathrm{b}}-\mathrm{H}}$ using STEX. The results are shown in Fig. 4 and compared to the $\mathrm{N}_{2}$ spectrum computed by the same method.

As $\mathrm{H}^{+}$approaches the fragment $\mathrm{N}_{2}$, two main effects can be noted. The first effect is that, while the values of the ionization thresholds (see IP curve in Fig. 2) increase as $R_{\mathrm{N}_{\mathrm{b}}-\mathrm{H}}$ decreases, the value of the STEX $\pi^{*}$ orbital energy $\left(\varepsilon_{\pi^{*}}\right)$ decreases instead (see also Fig. 5) and then the position of the lowest band shows a limited shift in $\mathrm{N}_{2} \mathrm{H}^{+}$compared to $\mathrm{N}_{2}$. Such blue shift increases monotonously as the nitrogen-proton distance decreases, it is well appreciable at a distance well above the $\mathrm{N}_{\mathrm{b}}-\mathrm{H}$ bond length
Table 2 Assignment of $\mathrm{N}_{2} \mathrm{H}^{+}$spectrum at RASPT2 level of theory, along with excitation energies and oscillator strength $(f)$; the correspondent full spectrum is shown in Fig. 1. $1 \sigma$ corresponds to the core orbital of atom $N_{b}$ and $2 \sigma$ to that of atom $\mathrm{N}_{\mathrm{a}}$. The theoretical energies were shifted by $-2.2 \mathrm{eV}$ in order to match the experimental spectrum in Fig. 1

\begin{tabular}{|c|c|c|}
\hline Ex. ener. (eV) & $f$ & [CI weight (\%)] assignment \\
\hline 401.49 & $1.20 \times 10^{-1}$ & {$[86] 1 \sigma^{-1} 2 \pi$} \\
\hline 401.72 & $1.06 \times 10^{-1}$ & {$[55] 2 \sigma^{-1} 2 \pi /[25] 2 \sigma^{-1} 1 \pi^{-1} 2 \pi^{2}$} \\
\hline 407.09 & $1.44 \times 10^{-2}$ & {$[77] 1 \sigma^{-1} \sigma_{\mathrm{H}}$} \\
\hline 407.22 & $9.35 \times 10^{-3}$ & {$[75] 2 \sigma^{-1} \sigma_{H}$} \\
\hline 410.01 & $1.20 \times 10^{-3}$ & {$[78] 1 \sigma^{-1} 3 \mathrm{~s} \sigma$} \\
\hline 410.48 & $6.99 \times 10^{-5}$ & {$[73] 2 \sigma^{-1} 3 \mathrm{~s} \sigma$} \\
\hline 410.93 & $2.78 \times 10^{-3}$ & {$[80] 2 \sigma^{-1} 1 \pi^{-1} 2 \pi^{2}$} \\
\hline 412.54 & $9.10 \times 10^{-4}$ & [78] $1 \sigma^{-1} 5 \sigma^{-1} 2 \pi^{2}$ \\
\hline 412.59 & $1.80 \times 10^{-2}$ & {$[61] 1 \sigma^{-1} 3 p \pi /[23] 1 \sigma^{-1} 4 p \pi$} \\
\hline 412.9 & $5.30 \times 10^{-4}$ & {$[71] 1 \sigma^{-1} 3 p \sigma$} \\
\hline 413.01 & $5.28 \times 10^{-5}$ & {$[80] 1 \sigma^{-1} 1 \pi^{-1} 2 \pi^{2}$} \\
\hline 413.21 & $4.71 \times 10^{-3}$ & $\begin{array}{l}{[17] 2 \sigma^{-1} 3 \mathrm{p} \pi /[17] 2 \sigma^{-1} 4 \mathrm{p} \pi} \\
{[18] 2 \sigma^{-1} 1 \pi^{-1} 2 \pi 3 \mathrm{p} \pi} \\
{[15] 2 \sigma^{-1} 1 \pi^{-1} 2 \pi 4 \mathrm{p} \pi}\end{array}$ \\
\hline 413.55 & $8.08 \times 10^{-3}$ & {$[67] 2 \sigma^{-1} 3 \mathrm{p} \sigma /[12] 2 \sigma^{-1} 3 \mathrm{~s} \sigma$} \\
\hline 413.69 & $4.14 \times 10^{-5}$ & {$[75] 2 \sigma^{-1} 1 \pi^{-1} 2 \pi^{2}$} \\
\hline 414.26 & $1.67 \times 10^{-5}$ & [79] $1 \sigma^{-1} 4 \mathrm{~s} \sigma$ \\
\hline 414.71 & $1.52 \times 10^{-3}$ & $80] 2 \sigma^{-1} 4 \mathrm{~s} \sigma$ \\
\hline 415.28 & $4.19 \times 10^{-3}$ & {$[57] 1 \sigma^{-1} 5 \mathrm{p} \pi /[18] 1 \sigma^{-1} 4 \mathrm{p} \pi$} \\
\hline 415.35 & $9.99 \times 10^{-4}$ & [83] $2 \sigma^{-1} 5 \sigma^{-1} 2 \pi$ \\
\hline 415.96 & $1.42 \times 10^{-2}$ & $\begin{array}{l}{[21] 2 \sigma^{-1} 3 \mathrm{p} \pi /[17] 2 \sigma^{-1} 4 \mathrm{p} \pi} \\
{[20] 2 \sigma^{-1} 1 \pi^{-1} 2 \pi 3 \mathrm{p} \pi} \\
{[18] 2 \sigma^{-1} 1 \pi^{-1} 2 \pi 4 \mathrm{p} \pi}\end{array}$ \\
\hline 416.59 & $6.24 \times 10^{-3}$ & $\begin{array}{l}{[45] 1 \sigma^{-1} 4 \mathrm{p} \pi /[27] 1 \sigma^{-1} 5 \mathrm{p} \pi} \\
13] 1 \sigma^{-1} 3 \mathrm{p} \pi\end{array}$ \\
\hline 417.03 & $8.04 \times 10^{-3}$ & [26] $2 \sigma^{-1} 1 \pi^{-1} 2 \pi^{2} /[11] 2 \sigma^{-1} 2 \pi$ \\
\hline 417.35 & $1.26 \times 10^{-4}$ & $\begin{array}{l}{[27] 2 \sigma^{-1} 5 \mathrm{p} \pi} \\
27] 2 \sigma^{-1} 1 \pi^{-1} 2 \pi 5 \mathrm{p} \pi\end{array}$ \\
\hline 417.96 & $3.93 \times 10^{-5}$ & [74] $2 \sigma^{-1} 1 \pi^{-1} \sigma_{\mathrm{H}} 2 \pi$ \\
\hline 418.08 & $7.16 \times 10^{-3}$ & [57] $1 \sigma^{-1} 1 \pi^{-1} 2 \pi^{2}$ \\
\hline 418.43 & $7.27 \times 10^{-5}$ & [89] $1 \sigma^{-1} 1 \pi^{-1} \sigma_{\mathrm{H}} 2 \pi$ \\
\hline 418.82 & $7.01 \times 10^{-3}$ & $\begin{array}{l}\text { [38] } 2 \sigma^{-1} 6 \mathrm{p} \pi \\
{[36] 2 \sigma^{-1} 1 \pi^{-1} 2 \pi 6 \mathrm{p} \pi}\end{array}$ \\
\hline 419.18 & $8.03 \times 10^{-4}$ & {$[65] 1 \sigma^{-1} 5 \sigma^{-1} \sigma_{\mathrm{H}} 2 \pi$} \\
\hline 421.50 & - & $2 \sigma^{-1}$ \\
\hline 421.57 & - & $1 \sigma^{-1}$ \\
\hline
\end{tabular}

ad reaches a maximum value of about $1 \mathrm{eV}$ at the equilibrium geometry, in good agreement with the experimental results in the first panel of Fig. 4. For values of $R_{\mathrm{N}_{\mathrm{b}}-\mathrm{H}}<3 \AA$ the effect of symmetry breaking induced by the presence of the proton becomes evident also in the separation of the two contributions (green for $\mathrm{N}_{\mathrm{a}}$ and red for $\mathrm{N}_{\mathrm{b}}$ ) to the first band. The second contribution, shifted by $0.13 \mathrm{eV}$ at the equilibrium geometry and with lower intensity, appears as a robust shoulder of the first peak. These results suggested that the two bands could hardly be resolved experimentally since for $\mathrm{N}_{2} \mathrm{H}^{+}$a rather "soft" bending mode is present with energy $<0.1 \mathrm{eV},{ }^{53}$ see further the vibrational analysis given in Section 4.6. The second effect is seen in the high energy region of the $\mathrm{N}_{2} \mathrm{H}^{+}$spectrum, where the weak bands of $\mathrm{N}_{2}$ seem to acquire intensity by the interaction with the proton and some of them, especially that around $413 \mathrm{eV}$ in the spectrum at the equilibrium geometry, show a striking energy variation versus $R_{\mathrm{N}_{\mathrm{b}}-\mathrm{H}}$.

Fig. 5 shows the shifted core ionization potential (IP) and the lowest four $(2 \pi, 6 \sigma, 7 \sigma, 3 \pi)$ STEX virtual orbital energies $(\varepsilon)$, for the $1 \sigma$ excitation channel, as a function of $R_{\mathrm{N}_{\mathrm{b}}-\mathrm{H}}$. At equilibrium geometry the LUMO (blue line) corresponds to the orbital $2 \pi\left(\pi^{*}\right)$ related to the $1 \pi_{\mathrm{g}}$ orbital of $\mathrm{N}_{2}$, while the 


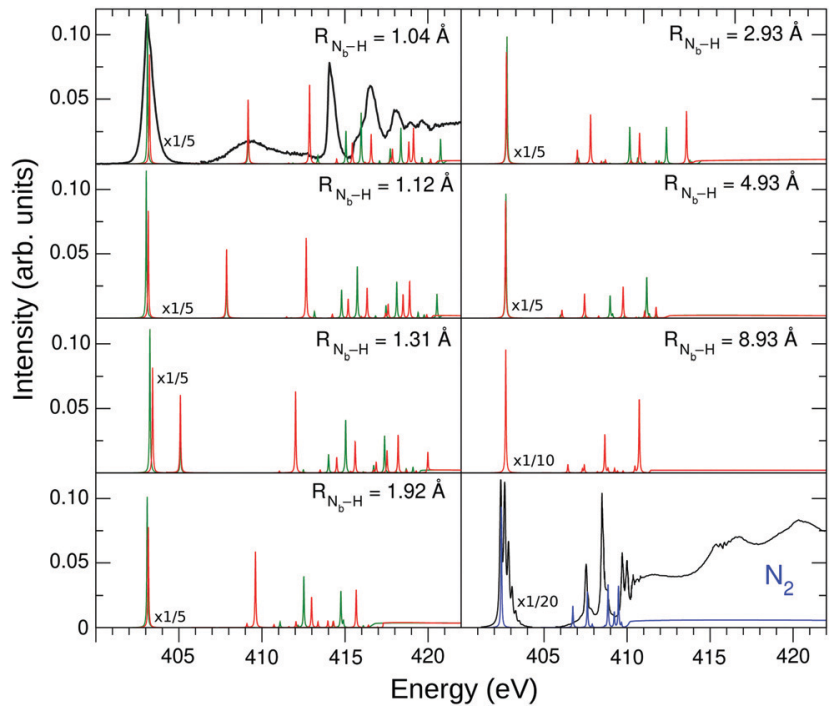

Fig. 4 STEX $\mathrm{N}$ K-edge photoabsorption spectrum of $\mathrm{N}_{2} \mathrm{H}^{+}$at different geometries, compared to that of $N_{2}$. The green bars represent the $N_{a}$ contribution to the spectrum, while the $N_{b}$ contribution is shown in red. The experimental spectra are represented by the black curves, which were arbitrary shifted to match the theoretical $\pi^{\star}$ peak. The experimental $\pi^{\star} N_{2}$ spectrum was obtained as described in Section 2, while the region above $405 \mathrm{eV}$ was taken from ref. 52.

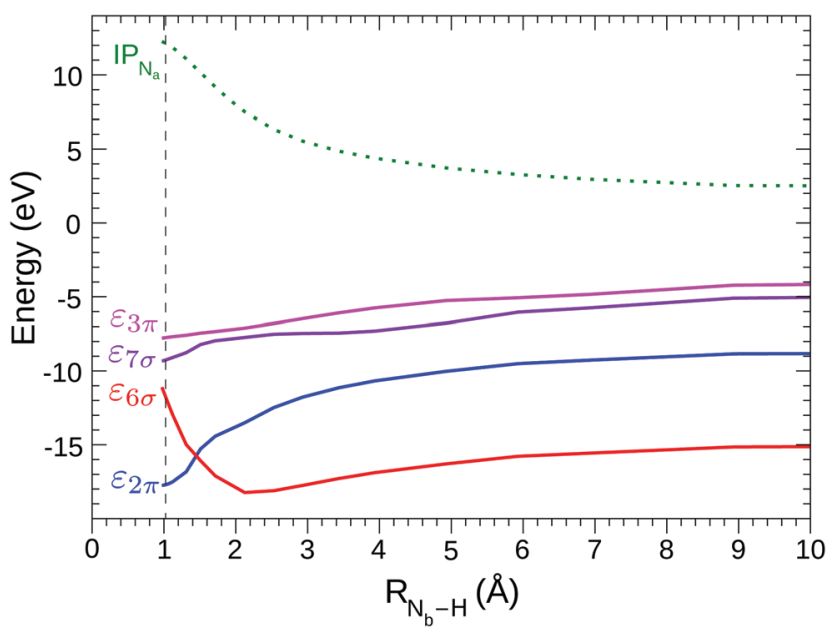

Fig. 5 STEX energies of the four lowest virtual orbitals ( $1 \sigma$ excitation channel) vs. the distance $\mathrm{N}_{\mathrm{b}}-\mathrm{H}$; the label $(\mathrm{H})$ of the lowest virtual orbital of $\sigma$ symmetry (red line) underlines the localization of the orbital. The green dashed line shows the $N_{a}$ core ionization threshold shifted by $-409 \mathrm{eV}$. The equilibrium value of $R_{N_{\mathrm{b}}-\mathrm{H}}$ is $1.0 \AA$, indicated by the vertical dashed line.

LUMO+1 $(6 \sigma$, red line) is the orbital with a clear localization around the proton and actually, both in energy and in form, it is similar to the hydrogenic orbital 1s. The increase of IP, as the proton approaches the $\mathrm{N}_{2}$ fragment, is due to the repulsive interaction between the charge of the proton and that of the core hole; conversely, the interaction between the proton and the electron in the $\pi^{*}$ orbital is attractive as shown by the blue curve in Fig. 5. It is somewhat surprising that, despite the different spatial distribution of the core hole and the $\pi^{*}$ orbital, the variation, in inverted sign, of IP and $\pi^{*}$ is very similar, so that, as already commented observing the spectra in Fig. 4, the position of the $\pi^{*}$ band varies little with $R_{\mathrm{N}_{\mathrm{b}}-\mathrm{H}}$. The trend of $\varepsilon_{6 \sigma}$ is completely different from that of $\varepsilon \pi^{*}$ for small $R_{\mathrm{N}_{\mathrm{b}}-\mathrm{H}}$ values since, in this case, the approaching fragment $\mathrm{H}$ is not charged and the interaction with the charge distribution of the other fragment is more complex, also including a short-range repulsive component. As a consequence of the trend of the red curve $\left(\varepsilon_{6 \sigma}\right)$ compared to the green dashed one (IP), the energy for the core excitation to such level, which is in fact a charge transfer excitation, is particularly mobile with $R_{\mathrm{N}_{\mathrm{b}}-\mathrm{H}}$, as already observed in Fig. 4 . The trend of the $\varepsilon_{6 \sigma}$ curve, when compared to that of $\mathrm{IP}_{\mathrm{N}_{\mathrm{a}}}$, also suggests that the $1 \sigma \rightarrow 6 \sigma$ excited state, reached by vertical transition, has a decidedly dissociative character. RASPT2 calculations for the two lowest excited states confirm that, see Fig. S1 in the ESI. $\dagger$

\section{5 $\mathrm{N}_{2} \mathrm{H}^{+}$photoabsorption spectrum at equilibrium geometry}

Fig. 1 (lower panel) presents the STEX spectrum of $\mathrm{N}_{2} \mathrm{H}^{+}$at the equilibrium geometry; the insert shows the high energy bands increased by a factor of 3. The RASPT2 results (excitation energies in eV, oscillator strengths, symmetry labels, and configurational expansion) for the bound excited states of $\mathrm{N}_{2} \mathrm{H}^{+}$are collected in Fig. 1 (top panel) and Table 2.

We recall here that while STEX represents the completion (or close to) of the 1-electron basis but with only a single configuration for excitations, RASPT2 is open ended towards full $\mathrm{N}$-electronic excitations, but, in practice, with limitations of the 1-particle basis set. For details in the parametrization we refer to the Computational section. One can note that the RASPT2 results of the two core hole spectra are quite similar to the STEX predictions for the $\pi^{*}$ and charge transfer excitations, while they are more spread for the upper irregular progression between 412 and $420 \mathrm{eV}$. The configurational composition of the charge transfer state is for both $\mathrm{N}_{\mathrm{a}}$ and $\mathrm{N}_{\mathrm{b}}$ transitions dominated by just one configuration, while one notes a difference for the $\pi^{*}$ states where a second, doubly excited component, appears for the excitation at $\mathrm{N}_{\mathrm{b}}$. Thus symmetry breaking appears to some extent also in the configurational representation of the two $\pi^{*}$ states. This type of difference is also reflected in the upper excitation region, $412-420 \mathrm{eV}$, where, similar to what was observed for $\mathrm{CO}^{+}$, where the localized $5 \sigma$ orbital leads to a stronger splitting at the carbon center, ${ }^{13}$ the configurational mixing seems more pronounced for the $\mathrm{N}_{\mathrm{b}}$ compared to the $\mathrm{N}_{\mathrm{a}}$ transitions. Also the charge transfer excitation can involve a concomitant extra excitation, but these transitions (which would be the counterpart of the semi-internal transitions in open-shell cationic spectra ${ }^{12}$ ) appear high up close to the IP and with small oscillator strengths.

\subsection{Vibrational analysis of the $\pi^{*}$ band}

In order to characterize the profile of the first and most intense band of the experimental spectrum of $\mathrm{N}_{2} \mathrm{H}^{+}$in Fig. 1, a detailed vibrational band analysis is called for. The band shows on the left side a rising slope, reaching maximum at $401.6 \mathrm{eV}$, and a more slowly descending slope on the right side, exhibiting a shoulder at $401.8 \mathrm{eV}$. We note that the low-energy side of the 
maximum shows an uncharacteristic rising slope. The question is if the band shape is simply due to the presence of two distinct $\pi^{*}$ excitations or it is also due to a vibrational structure more complex than that of $\mathrm{N}_{2}$. We have then applied a multidimensional Franck-Condon analysis to explore the origin of this band shape using DFT theory as described in Section 3.2 and in the ESI. $\dagger$

The validation of the method was performed by simulating the one-dimensional vibrational $\pi^{*}$ band of $\mathrm{N}_{2}$ and the best result, see Fig. 6, was obtained using the BLYP functional. It is here interesting to note that the vibrational profiles of the two $\pi^{*}$ excitations of $\mathrm{N}_{2} \mathrm{H}^{+}$in Fig. 6, obtained by considering only the two stretching modes, are quite different; $\mathrm{N}_{\mathrm{a}}$ shows a more restricted progression, while $\mathrm{N}_{\mathrm{b}}$, as well as $\mathrm{N}_{2}$ shows a more extended one. The difference in these extensions reflects a difference in the excited state energy gradients, which also is a measure of symmetry breaking. The compound band shape (brown line in Fig. 6) is the superposition of the two vibrational structures based on the relative electronic transition energies and intensities. From Fig. 6 it appears clearly how the simulated vibrational profile for $\mathrm{N}_{2}$ is in quantitative agreement with the experimental one, while for $\mathrm{N}_{2} \mathrm{H}^{+}$it is not possible to obtain even a qualitative agreement on the low-energy side of the band,
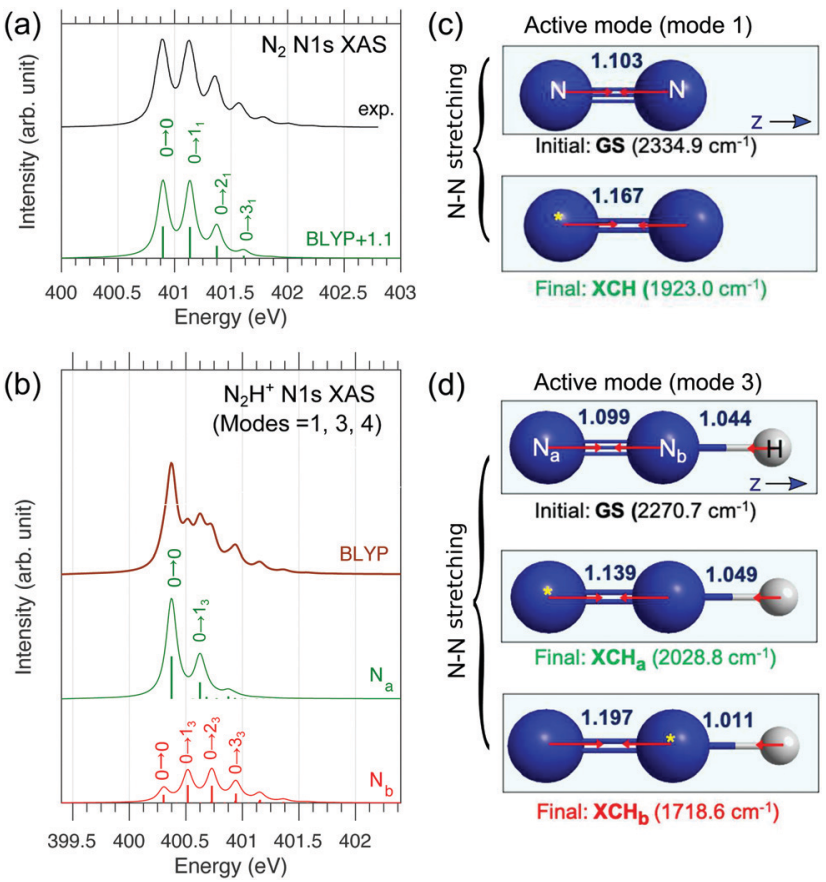

Fig. 6 Theoretical simulation of the vibrationally-resolved N1s $\rightarrow \pi^{\star}$ XAS band of (a) $\mathrm{N}_{2}$ and (b) $\mathrm{N}_{2} \mathrm{H}^{+}$computed by the BLYP and Duschinsky rotation method. For $\mathrm{N}_{2} \mathrm{H}^{+}$only three modes $(1,3,4$; see Fig. S4, ESI $\uparrow$ ) are considered; dominant peaks were assigned. In panel $b$, atom-specific stick spectra are shown (already scaled with electronic oscillator strengths, see $\mathrm{ESI} \dagger$ ), and summation gives the total spectrum (brown). For $\mathrm{N}_{2}$, an ad hoc uniform shift of $1.1 \mathrm{eV}$ was added to the theoretical spectrum to better compare with experiment. ( $c$ and $d$ ) Linear structures of the ground and $\mathrm{XCH}$ states (bond lengths are in $\AA$; yellow stars denote the core holes) with active vibrational modes illustrated. For $\mathrm{N}_{2} \mathrm{H}^{+}$, the $\mathrm{N}-\mathrm{N}$ stretching mode (mode 3 ) is dominantly active. for any relative alignment of the two calculated vibrational structures. This could be an indication of vibrational excitation in the initial state as was also observed for $\mathrm{N}_{2}{ }^{+12}$ or/and to a broadening of the vibrational profile due to the bending mode.

The labels reported on the single peaks of the $\mathrm{N}_{\mathrm{a}}$ and $\mathrm{N}_{\mathrm{b}}$ contributions (Fig. 6) show that the only mode activated by the excitation seems to be the $\mathrm{N}-\mathrm{N}$ stretching assuming the linear geometry of the molecule. However in the initial and final states the bond angle changes from 180 to 120 degrees; this underlines how the anharmonicity of the bending motion must be explicitly considered. As discussed in Section 3 and in the ESI, $\dagger$ we have adopted a simplified 1D model for the description of the bending motion based on the decoupling of this mode from the other ones. This was accomplished by computing the potential energy curves for the core-excited state $\pi_{x z}{ }^{*}$ along the $x z$ bending mode, which has a double-well shape (Fig. S2, ESI $\dagger$ ). Thus, the total FC factors are given by

$$
\Omega_{\mathrm{nlm}}^{\mathrm{tot}}=\Omega_{\mathrm{n}}^{\mathrm{bend}} \Omega_{\mathrm{lm}}^{\mathrm{strs}},
$$

with the $x z$ bending $\left(\Omega_{\mathrm{n}}^{\text {bend }}\right)$ and stretching $\left(\Omega_{\operatorname{lm}}^{\text {strs }}\right)$ FC factors. Here $n$ is the quantum number for this bending mode, and 1 and $\mathrm{m}$ are for the $\mathrm{N}-\mathrm{N}$ and $\mathrm{N}-\mathrm{H}$ stretching modes, respectively. Note that the $y z$ bending mode is inactive since the potential energy surface (PES) of the $\pi_{x z}{ }^{*}$ core-excited state is similar to the ground-state PES except for a vertical shift. ${ }^{54}$ As previously indicated, by combining energies and FC factors calculated separately for the two kinds of vibrations, we finally obtained the $\mathrm{N}_{\mathrm{a}}$ and $\mathrm{N}_{\mathrm{b}}$ vibrational bands shown in Fig. 7. These appear quite different from those in Fig. 6 due to the contribution of

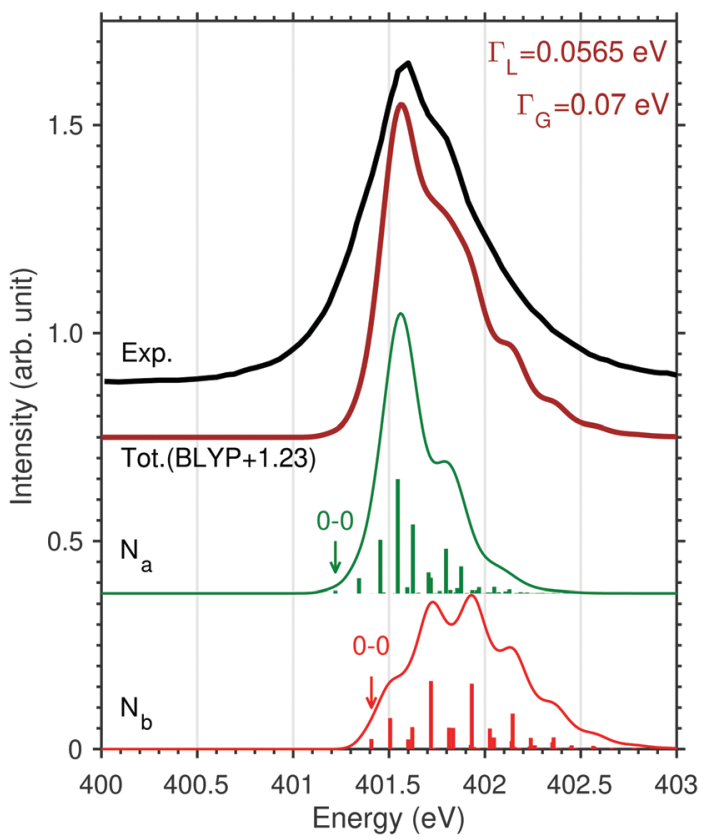

Fig. 7 FC factors computed at the BLYP level for the N1s $\rightarrow \pi^{\star}$ excitations of $\mathrm{N}_{2} \mathrm{H}^{+}$combing two stretching and one bending modes: $\mathrm{N}_{a}$ (green), $\mathrm{N}_{b}$ (red). An uniform ad hoc shift of $+1.23 \mathrm{eV}$ was applied to the total theoretical profile (brown line) to better compare with experiment (black line). 
the FC factors of the non-harmonic bending wave functions. In presence of the double well potential with a relatively low energy barrier (Fig. S2, ESI $\dagger$ ), the wave function $\Psi_{n_{\text {bend }}}$ with $n$ starting from 3, shows an appreciable tunnel effect which means an appreciable overlap with the ground vibrational wave function of the initial state. The short $\left(\mathrm{N}_{\mathrm{a}}\right)$ and long $\left(\mathrm{N}_{\mathrm{b}}\right)$ progression in Fig. 7 show the expected Poisson respectively Gaussian like distributions of peaks.

The superposition of the two vibrational progressions, obtained by using the experimental line broadening value given in the Experimental section, can offer, see Fig. 7, both an explanation of the higher energy slope including the shoulder at $401.8 \mathrm{eV}$ and the slowly rising low-energy part of the band. It is also clear though that the superposition is delicately dependent on the electronic energy separation of the two $\pi^{*}$ excited states. All these effects are subtle and can probably only be resolved in simple protonated species as $\mathrm{N}_{2} \mathrm{H}^{+}$studied here, whereas in XAS of protonated peptides or proteins ${ }^{55-58}$ these features would probably not be observed.

\section{Summary}

By comparison of diazynium to dinitrogen and the dinitrogen cation, we have explored the effect of symmetry breaking by protonation of homonuclear diatomic molecules on their NEXAFS spectra. Different from ionization, protonation of $\mathrm{N}_{2}$ changes the charge state without changing the number of valence electrons, but breaks inversion symmetry instead. As for the dinitrogen cation, the rich nitrogen $\mathrm{K}$ edge spectrum is extended by a factor of two for $\mathrm{N}_{2} \mathrm{H}^{+}$over dinitrogen by the role of the positive charge in the initial state. Again similar to $\mathrm{N}_{2}{ }^{+}$, the role of electron correlation, as predicted by RASSCF calculations, is significant in the high-energy part of the spectrum of $\mathrm{N}_{2} \mathrm{H}^{+}$ as well.

An instructive comparison of the appearance of cationic NEXAFS spectra can be made when the studied species have been charged by the addition of a proton versus the case when the molecule has been ionized through removal of an electron from the HOMO. Our recent studies of $\mathrm{N}_{2}^{+12}$ and $\mathrm{CO}^{+13}$ indicated widely different and conspicuous features of the X-ray absorption spectra as compared to the corresponding neutral species. In particular, a three-fold division of the spectra could be made, none with correspondence in NEXAFS spectra of neutral molecules; at lowest transition energy, by an excitation to the valence orbital hole that was created in the ionization process, so creating a bare core hole state with correspondence to X-ray photoelectron spectroscopy but reached from a different initial state and following different selection rules; a second part showing spin-coupled split bands corresponding to core-valence transitions; and a remaining long and weak progression containing a manifold of peaks with irregular appearance manifesting highly excited states with a character of strong electron correlation. In case of protonated closed shell species, in place of the core $\rightarrow$ SOMO transition, we can have a charge transfer transition towards the proton forming a hydrogen, as we can conclude for $\mathrm{N}_{2} \mathrm{H}^{+}$molecule at the equilibrium geometry.

Because inversion symmetry is broken in $\mathrm{N}_{2} \mathrm{H}^{+}$, there is no inversion-symmetry forbidden transition as in the dinitrogen molecule or molecular ion. This gives rise to two distinct $1 \mathrm{~s} \sigma$ to $\pi^{*}$ transitions in the spectrum of $\mathrm{N}_{2} \mathrm{H}^{+}$, with substantial broadening because of complex vibrational excitation in the bent core excited state. Our detailed computational analysis links the transition in dinitrogen and $\mathrm{N}_{2} \mathrm{H}^{+}$, and shows that the presence of the proton in $\mathrm{N}_{2} \mathrm{H}^{+}$opens the corresponding symmetry-forbidden excitation in $\mathrm{N}_{2}$. In order to analyze more in detail the complex shape of the experimental $\pi^{*}$ band, a multimode vibrational simulation was performed. It was predicted that the $\mathrm{N}-\mathrm{N}$ stretch excitation in $\mathrm{N}_{2}$ is reduced for the terminal $\mathrm{N}_{\mathrm{a}}-\pi^{*}$ excitation while being further prolonged for the $\mathrm{N}_{\mathrm{b}}-\pi^{*}$ excitation and that the bending mode causes a substantial broadening of the band.

We have also computationally traced the appearance of the NEXAFS spectrum for a proton approaching from infinity to the $\mathrm{N}_{2}$ molecule up to the equilibrium geometry of $\mathrm{N}_{2} \mathrm{H}^{+}$. The separation of the two $\pi^{*}$ peaks and the development of the electronic structure and of the charge transfer excitation show smooth dependence on the proton position. An interesting feature of partial localization of the nitrogen core orbitals with respect to proton distance could be unraveled. This localization shows a strong, non-monotonous, variation with proton distance qualitatively independent of the theory method applied.

In summary, as in the case of NEXAFS spectra of molecular cations obtained by ionization, ${ }^{12,13}$ our study on the $\mathrm{N}_{2} \mathrm{H}^{+}$ spectrum unravels new and unexplored effects also when the cation is obtained by molecular protonation. These spectra may also inherently contain rich information on chemistry in terms of electron localization, chemical bonding, and structural conformation when applied to larger molecular cations.

\section{Author contributions}

R. C. C., W. H. and V. C. performed all simulations and theoretical analysis, and prepared the text of manuscript and figures with J. T. L. and B. v. I; L. K., R. L., M. K., C. B., M. T., V. Z.-B., J. S., S. L. S., B. v. I., J. T. L., J.-E. R. suggested and planned the experiment, collected the data and carried out the data analysis; J. T. L., V. Z.-B., B. v. I. developed and built the experimental setup; H. A., V. C. took main responsibility for the theoretical modelling, results discussion and the writing of the paper, in which all authors contributed. All authors reviewed the manuscript.

\section{Conflicts of interest}

There are no conflicts to declare.

\section{Acknowledgements}

The beamtime for this project was granted at BESSY II beamline UE52-PGM and Ion Trap endstation, operated by HelmholtzZentrum Berlin. The simulations were performed on resources 
provided by the Swedish National Infrastructure for Computing (SNIC) at the National Supercomputer Center (NSC), through the project "Multiphysics Modeling of Molecular Materials" (SNIC 2019/2-41 and 2020/3-29), and the Shanghai Supercomputer Center. This project has received funding from the European Union's Horizon 2020 research and innovation programme under grant agreement no. 730872 and by the German Federal Ministry of Education and Research (BMBF) through Grant No. BMBF05K16Vf2. R. L. and J.-E. R. acknowledge funding from the Swedish Research Council, contract 637-2014-6929, and 201404518, respectively. W. H. was supported by National Natural Science Foundation of China (11774174, 21703105).

\section{References}

1 P. S. Bagus and H. F. Schaefer, J. Chem. Phys., 1972, 56, 224-226.

2 F. Gel'mukhanov and H. Ågren, Phys. Rep., 1999, 312, $87-330$.

3 D. Rolles, M. Braune, S. Cvejanović, O. Geßner, R. Hentges, S. Korica, B. Langer, T. Lischke, G. Prümper and A. Reinköster, et al., Nature, 2005, 437, 711-715.

4 M. F. Kling, C. Siedschlag, A. J. Verhoef, J. I. Khan, M. Schultze, T. Uphues, Y. Ni, M. Uiberacker, M. Drescher, F. Krausz and M. J. J. Vrakking, Science, 2006, 312, 246-248.

5 F. Martn, J. Fernández, T. Havermeier, L. Foucar, T. Weber, K. Kreidi, M. Schöffler, L. Schmidt, T. Jahnke, O. Jagutzki, A. Czasch, E. P. Benis, T. Osipov, A. L. Landers, A. Belkacem, M. H. Prior, H. Schmidt-Böcking, C. L. Cocke and R. Dörner, Science, 2007, 315, 629-633.

6 M. S. Schöffler, J. Titze, N. Petridis, T. Jahnke, K. Cole, L. P. H. Schmidt, A. Czasch, D. Akoury, O. Jagutzki, J. B. Williams, N. A. Cherepkov, S. K. Semenov, C. W. McCurdy, T. N. Rescigno, C. L. Cocke, T. Osipov, S. Lee, M. H. Prior, A. Belkacem, A. L. Landers, H. Schmidt-Böcking, T. Weber and R. Dörner, Science, 2008, 320, 920-923.

7 K. Hirsch, J. T. Lau, P. Klar, A. Langenberg, J. Probst, J. Rittmann, M. Vogel, V. Zamudio-Bayer, T. Möller and B. von Issendorff, J. Phys. B: At., Mol. Opt. Phys., 2009, 42, 154029.

8 V. Zamudio-Bayer, K. Hirsch, A. Langenberg, A. aawicki, A. Terasaki, B. V. Issendorff and J. T. Lau, J. Chem. Phys., 2015, 143, 244318.

9 J.-P. Mosnier, E. T. Kennedy, P. van Kampen, D. Cubaynes, S. Guilbaud, N. Sisourat, A. Puglisi, S. Carniato and J.-M. Bizau, Phys. Rev. A, 2016, 93, 061401.

10 S. Klumpp, A. A. Guda, K. Schubert, K. Mertens, J. Hellhund, A. Müller, S. Schippers, S. Bari and M. Martins, Phys. Rev. A, 2018, 97, 033401.

11 S. Bari, L. Inhester, K. Schubert, K. Mertens, J. O. Schunck, S. Dörner, S. Deinert, L. Schwob, S. Schippers, A. Müller, S. Klumpp and M. Martins, Phys. Chem. Chem. Phys., 2019, 21, 16505-16514.

12 R. Lindblad, L. Kjellsson, R. C. Couto, M. Timm, C. Bülow, V. Zamudio-Bayer, M. Lundberg, B. von Issendorff, J. T. Lau, S. L. Sorensen, V. Carravetta, H. Ågren and J.-E. Rubensson, Phys. Rev. Lett., 2020, 124, 203001.
13 R. C. Couto, L. Kjellsson, H. Ågren, V. Carravetta, S. L. Sorensen, M. Kubin, C. Bülow, M. Timm, V. ZamudioBayer, B. von Issendorff, J. T. Lau, J. Söderström, J.-E. Rubensson and R. Lindblad, Phys. Chem. Chem. Phys, 2020, 22, 16215-16223.

14 S. Carniato, J.-M. Bizau, D. Cubaynes, E. T. Kennedy, S. Guilbaud, E. Sokell, B. McLaughlin and J.-P. Mosnier, Atoms, 2020, 8, 67.

15 P. Caselli, P. C. Myers and P. Thaddeus, Astrophys. J., 1995, 455, L77-L80.

16 W. D. Geppert and M. Larsson, Chem. Rev., 2013, 113, 8872-8905.

17 J. C. Owrutsky, C. S. Gudeman, C. C. Martner, L. M. Tack, N. H. Rosenbaum and R. J. Saykally, J. Chem. Phys., 1986, 84, 605-617.

18 T. Amano, T. Hirao and J. Takano, J. Mol. Spectrosc., 2005, 234, 170-175.

19 M. Niemeyer, K. Hirsch, V. Zamudio-Bayer, A. Langenberg, M. Vogel, M. Kossick, C. Ebrecht, K. Egashira, A. Terasaki, T. Möller, B. v. Issendorff and J. T. Lau, Phys. Rev. Lett., 2012, 108, 057201.

20 V. Zamudio-Bayer, K. Hirsch, A. Langenberg, M. Niemeyer, M. Vogel, A. aawicki, A. Terasaki, J. T. Lau and B. von Issendorff, Angew. Chem., Int. Ed., 2015, 54, 4498-4501.

21 V. Zamudio-Bayer, K. Hirsch, A. Langenberg, M. Kossick, A. aawicki, A. Terasaki, B. v. Issendorff and J. T. Lau, J. Chem. Phys., 2015, 142, 234301.

22 S. Schippers, A. L. D. Kilcoyne, R. A. Phaneuf and A. Müller, Contemp. Phys., 2016, 57, 215-229.

23 M. Meuwly, S. A. Nizkorodov, J. P. Maier and E. J. Bieske, J. Chem. Phys., 1996, 104, 3876-3885.

24 M. R. Weiss, R. Follath, K. J. S. Sawhney and T. Zeschke, Nucl. Instrum. Methods Phys. Res., 2001, 467-468, 482-484.

25 S. Forsen and B. Roos, Chem. Phys. Lett., 1970, 6, 128-132.

26 K. Vasudevan, S. Peyerimhoff and R. Buenker, Chem. Phys., 1974, 5, 149-165.

27 K. C. Sears, J. W. Ferguson, T. J. Dudley, R. S. Houk and M. S. Gordon, J. Phys. Chem. A, 2008, 112, 2610-2617.

28 P. Å. Malmqvist, K. Pierloot, A. R. M. Shahi, C. J. Cramer and L. Gagliardi, J. Chem. Phys., 2008, 128, 204109.

29 B. O. Roos, R. Lindh, P.-Å. Malmqvist, V. Veryazov and P.-O. Widmark, J. Phys. Chem. A, 2004, 108, 2851-2858.

30 H. Ågren, V. Carravetta, O. Vahtras and L. G. Pettersson, Theor. Chem. Acc., 1997, 97, 14-40.

31 H. J. A. Jensen, P. Jørgensen and H. Ågren, J. Chem. Phys., 1987, 87, 451-466.

32 H. Ågren, A. Flores-Riveros and H. Jensen, Phys. Scr., 1989, 40, 745-750.

33 P. Å. Malmqvist, A. Rendell and B. O. Roos, J. Phys. Chem., 1990, 94, 5477-5482.

34 I. F. Galván, M. Vacher, A. Alavi, C. Angeli, F. Aquilante, J. Autschbach, J. J. Bao, S. I. Bokarev, N. A. Bogdanov and R. K. Carlson, et al., J. Chem. Theory Comput., 2019, 15, 5925-5964.

35 L. S. Cederbaum, W. Domcke and J. Schirmer, Phys. Rev. A, 1980, 22, 206-222. 
36 M. G. Delcey, L. K. Sørensen, M. Vacher, R. C. Couto and M. Lundberg, J. Comput. Chem., 2019, 40, 1789-1799.

37 K. Aidas, C. Angeli, K. L. Bak, V. Bakken, R. Bast, L. Boman, O. Christiansen, R. Cimiraglia, S. Coriani, P. Dahle, E. K. Dalskov, U. Ekström, T. Enevoldsen, J. J. Eriksen, P. Ettenhuber, B. Fernández, L. Ferrighi, H. Fliegl, L. Frediani, K. Hald, A. Halkier, C. Hättig, H. Heiberg, T. Helgaker, A. C. Hennum, H. Hettema, E. Hjertens, S. Høst, I.-M. Høyvik, M. F. Iozzi, B. Jansík, H. J. A. Jensen, D. Jonsson, P. Jørgensen, J. Kauczor, S. Kirpekar, T. Kjrgaard, W. Klopper, S. Knecht, R. Kobayashi, H. Koch, J. Kongsted, A. Krapp, K. Kristensen, A. Ligabue, O. B. Lutns, J. I. Melo, K. V. Mikkelsen, R. H. Myhre, C. Neiss, C. B. Nielsen, P. Norman, J. Olsen, J. M. H. Olsen, A. Osted, M. J. Packer, F. Pawlowski, T. B. Pedersen, P. F. Provasi, S. Reine, Z. Rinkevicius, T. A. Ruden, K. Ruud, V. V. Rybkin, P. Sałek, C. C. M. Samson, A. S. de Merás, T. Saue, S. P. A. Sauer, B. Schimmelpfennig, K. Sneskov, A. H. Steindal, K. O. Sylvester-Hvid, P. R. Taylor, A. M. Teale, E. I. Tellgren, D. P. Tew, A. J. Thorvaldsen, L. Thøgersen, O. Vahtras, M. A. Watson, D. J. D. Wilson, M. Ziolkowski and H. Ågren, Wiley Interdiscip. Rev.: Comput. Mol. Sci., 2014, 4, 269-284.

38 T. H. Dunning, J. Chem. Phys., 1989, 90, 1007-1023.

39 R. A. Kendall, T. H. Dunning and R. J. Harrison, J. Chem. Phys., 1992, 96, 6796-6806.

40 M. Douglas and N. M. Kroll, Ann. Phys., 1974, 82, 89-155.

41 B. A. Hess, Phys. Rev. A, 1986, 33, 3742-3748.

42 N. Forsberg and P.-Å. Malmqvist, Chem. Phys. Lett., 1997, 274, 196-204.

43 G. Ghigo, B. O. Roos and P.-Å. Malmqvist, Chem. Phys. Lett., 2004, 396, 142-149.

44 P.-Å. Malmqvist and B. O. Roos, Chem. Phys. Lett., 1989, 155, 189-194.

45 P. Å. Malmqvist, B. O. Roos and B. Schimmelpfennig, Chem. Phys. Lett., 2002, 357, 230-240.
46 G. Tian, S. Duan, W. Hua and Y. Luo, DynaVib, version 1.0, Royal Institute of Technology, Sweden, 2012.

47 M. W. Schmidt, K. K. Baldridge, J. A. Boatz, S. T. Elbert, M. S. Gordon, J. H. Jensen, S. Koseki, N. Matsunaga, K. A. Nguyen, S. Su, T. L. Windus, M. Dupuis and J. A. Montgomery Jr, J. Comput. Chem., 1993, 14, 1347-1363.

48 M. S. Gordon and M. W. Schmidt, in Advances in electronic structure theory: GAMESS a decade later, ed. C. E. Dykstra, G. Frenking, K. S. Kim and G. E. Scuseria, Elsevier, Amsterdam, 2005, ch. 41, pp. 1167-1189.

49 A. D. Becke, Phys. Rev. A, 1988, 38, 3098.

50 W. Hua, G. Tian and Y. Luo, Phys. Chem. Chem. Phys., 2020, 22, 20014-20026.

51 F. Duschinsky, Acta Physicochim. URSS, 1937, 7, 551-566.

52 C. T. Chen, Y. Ma and F. Sette, Phys. Rev. A, 1989, 40, 6737-6740.

53 Y. Kabbadj, T. Huet, B. Rehfuss, C. Gabrys and T. Oka, J. Mol. Spectrosc., 1994, 163, 180-205.

54 J.-i. Adachi, N. Kosugi and A. Yagishita, J. Phys. B: At., Mol. Opt. Phys., 2005, 38, R127-R152.

55 D. Egorov, L. Schwob, M. Lalande, R. Hoekstra and T. Schlathölter, Phys. Chem. Chem. Phys., 2016, 18, 26213-26223.

56 S. Bari, D. Egorov, T. L. C. Jansen, R. Boll, R. Hoekstra, S. Techert, V. Zamudio-Bayer, C. Bülow, R. Lindblad, G. Leistner, A. aawicki, K. Hirsch, P. S. Miedema, B. von Issendorff, J. T. Lau and T. Schlathölter, Chem. - Eur. J., 2018, 24, 7631-7636.

57 D. Egorov, S. Bari, R. Boll, S. Dörner, S. Deinert, S. Techert, R. Hoekstra, V. Zamudio-Bayer, R. Lindblad, C. Bülow, M. Timm, B. von Issendorff, J. T. Lau and T. Schlathölter, J. Am. Soc. Mass Spectrom., 2018, 29, 2138-2151.

58 S. Dörner, L. Schwob, K. Atak, K. Schubert, R. Boll, T. Schlathölter, M. Timm, C. Bülow, V. Zamudio-Bayer, B. von Issendorff, J. T. Lau, S. Techert and S. Bari, J. Am. Soc. Mass Spectrom., 2021, 32, 670-684. 